\title{
Normal and inverted hysteresis in perovskite solar cells
}

\author{
George Alexandru Nemnes,, , 2, 田 Cristina Besleaga, ${ }^{3}$ Viorica Stancu, ${ }^{3}$ Lucia Nicoleta Leonat, ${ }^{3}$ \\ Lucian Pintilie, ${ }^{3}$ Kristinn Torfason, ${ }^{4}$ Marjan Ilkov, ${ }^{4,5}$ Andrei Manolescu, ${ }^{4}$ and Ioana Pintilie ${ }^{3}$, \\ ${ }^{1}$ University of Bucharest, Faculty of Physics, Materials and Devices for \\ Electronics and Optoelectronics Research Center, 077125 Magurele-Ilfov, Romania \\ ${ }^{2}$ Horia Hulubei National Institute for Physics and Nuclear Engineering, 07r126 Magurele-Ilfov, Romania \\ ${ }^{3}$ National Institute of Materials Physics, Magurele 077125, Ilfov, Romania \\ ${ }^{4}$ School of Science and Engineering, Reykjavik University, Menntavegur 1, IS-101 Reykjavik, Iceland \\ ${ }^{5}$ Icelandic Heart Association, Holtasmari 1, IS-201 Kopavogur, Iceland
}

\begin{abstract}
Hysteretic effects are investigated in perovskite solar cells in the standard $\mathrm{FTO} / \mathrm{TiO}_{2} / \mathrm{CH}_{3} \mathrm{NH}_{3} \mathrm{PbI}_{3-x} \mathrm{Cl}_{x}$ /spiro-OMeTAD/Au configuration. We report normal (NH) and inverted hysteresis (IH) in the $\mathrm{J}-\mathrm{V}$ characteristics occurring for the same device structure, the behavior strictly depending on the pre-poling bias. NH typically appears at pre-poling biases larger than the open circuit bias, while pronounced IH occurs for negative bias pre-poling. The transition from $\mathrm{NH}$ to $\mathrm{IH}$ is marked by a intermediate mixed hysteresis behavior characterized by a crossing point in the $\mathrm{J}-\mathrm{V}$ characteristics. The measured $\mathrm{J}-\mathrm{V}$ characteristics are explained quantitatively by the dynamic electrical model (DEM). Furthermore, the influence of the bias scan rate on the NH/IH hysteresis is discussed based on the time evolution of the non-linear polarization. Introducing a three step measurement protocol, which includes stabilization, pre-poling and measurement, we put forward the difficulties and possible solutions for a correct PCE evaluation.
\end{abstract}

\section{INTRODUCTION}

The dynamic hysteresis phenomena observed in the J$\mathrm{V}$ characteristics of perovskite solar cells (PSCs) fuels an ongoing debate about its origin, accurate determination of the photoconversion efficiency (PCE), and cell stability [1-4]. Several mechanisms have been proposed to explain the hysteretic behavior: ion migration [5 9], charge trapping and de-trapping [10, 11, ferroelectric polarization [12 15], capacitive effects [16 18], charge accumulation at the interfaces [19, 20], or unbalanced distribution of electrons and holes [21]. It is already established that the hysteresis is influenced by measurement settings such as the bias scan rate and range [5], but also by the cell pre-conditioning by voltage poling 22] and light soaking [23].

In the typical hysteresis the reverse-scan current, i.e. measured by reducing the voltage from open circuit bias $\left(V_{o c}\right)$ to short circuit, is larger than in the forward scan. During the reverse scan the cell behaves like a capacitor, releasing charge in the external circuit, and hence an excess of current is obtained, while during the forward scan the opposite situation occurs. We call this behavior normal hysteresis (NH). Recently an inverted hysteresis $(\mathrm{IH})$ was reported [24] in mixed-cation mixedhalide PSCs, and attributed to charge extraction barriers. It was further shown that methylammonium-leadiodide $\left(\mathrm{MAPbI}_{3}\right) \mathrm{PSCs}$ with $\mathrm{TiO}_{2}$ layer covered by a thin $\mathrm{Al}_{2} \mathrm{O}_{3}$ insulating shell also exhibits IH. In other studies the IH was explained in terms of ionic accumulation [25], and observed in aged samples with $\mathrm{Mo} / \mathrm{Ag}$ counter electrodes [26].

\footnotetext{
* nemnes@solid.fizica.unibuc.ro

† ioana@infim.ro
}

In this paper we report for the first time the presence of both $\mathrm{NH}$ and $\mathrm{IH}$ in the same sample, strictly depending on the applied pre-poling bias $\left(V_{p o l}\right): \mathrm{NH}$ is obtained for $V_{p o l}>V_{o c}$ and IH for $V_{p o l}<0$. For $0<V_{p o l}<V_{o c}$ the hysteresis loop has a crossing point where the forward and reverse characteristics meet. The experimental data are well reproduced by the dynamic electrical model which is summarized below. Then, we indicate a threestep J-V measurement protocol which is essential for a meaningful evaluation of the hysteretic effects. We discuss the pre-poling bias conditions for the realization of $\mathrm{NH}, \mathrm{IH}$ and intermediate mixed hysteresis. In connection with the measurement protocol, by controlling the switching between $\mathrm{NH}$ and $\mathrm{IH}$, we provide further insight into PSC operation but also a systematic assessment of the dynamic hysteresis, which is crucial for a proper evaluation of the PCE.

\section{THE DYNAMIC ELECTRICAL MODEL (DEM)}

Several models have been considered for the dynamic hysteresis: Initially a drift-diffusion approach has been formulated, accounting for ionic migration, electronic charge traps, and recombination centers [27], or coupled charge carriers and defect mediated ion motion including bias scan rate and pre-poling [28]. Then, an equivalent circuit model with constant capacitance was proposed [29]. The DEM, recently formulated by our group [30], is also based on an equivalent circuit, but includes a capacitor accounting for non-linear polarization effects as depicted in Fig. 1. The key assumption is that the slow process governing the time evolution of the non-linear polarization $P_{n l}$ can be described by a specific relaxation time $\tau$. Since $\tau$ is typically of the order of seconds DEM 


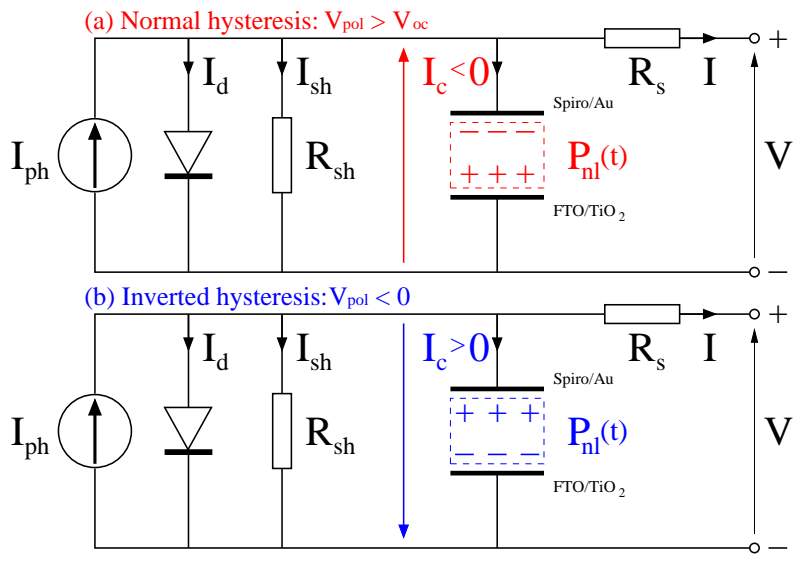

FIG. 1. Schematics of the dynamic electrical model for two different poling conditions: (a) $V_{p o l}>V_{o c}(\mathrm{NH})$ and (b) $V_{\text {pol }}<0(\mathrm{IH})$ induce opposite polarization orientations. The sign of $I_{c} \sim \partial P_{n l} / \partial t$ is indicated in both cases for a reverse scan starting from $V_{o c}$, after the poling step.

is compatible with ion migration and accumulation at the interfaces resulting in a surface polarization of MaxwellWagner-Sillars type. The time dependent J-V characteristics is determined by the coupled differential equations:

$$
\begin{aligned}
-R_{s} C_{0} \frac{\partial I}{\partial t} & =I_{s}\left(e^{\frac{q\left(V+I R_{s}\right)}{n k_{B} T}}-1\right) \\
& +\left(\frac{R_{s}}{R_{s h}}+1\right) I \\
& +\frac{V}{R_{s h}}+C_{0} \frac{\partial V}{\partial t}+\mathcal{A} \frac{\partial P_{n l}}{\partial t}-I_{p h} \\
\frac{\partial P_{n l}}{\partial t} & =\frac{P_{n l, \infty}\left(U_{c}(t)\right)-P_{n l}(t)}{\tau},
\end{aligned}
$$

with initial conditions $I(t=0)=I_{0}$ and $P_{n l}(t=0)=P_{0}$, which determines the $\mathrm{NH}$ or $\mathrm{IH}$ behavior. The current density $J$ is defined as the ratio between the current intensity $I$ and the device active area $\mathcal{A}$. The elements of the circuit are: the series resistance $R_{s}$, the shunt resistance $R_{s h}$, the diode ideality factor $n$, the diode saturation current $I_{s}$, the photogenerated current $I_{p h}$ and the geometric capacitance $C_{0}$. In contrast to the standard model with a constant capacitance, the time dependence of the non-linear polarization $P_{n l}$ obeys Eq. (2), with a constant relaxation time $\tau$, whereas the equilibrium polarization $P_{n l, \infty}=\left(U_{c} / V_{o c}\right) P_{\infty}$ depends on the voltage on the non-linear capacitor, $U_{c}=V+I R_{s}$, where $V$ is the external applied bias. The constant polarization $P_{\infty}$ corresponds to $U_{c}=V_{o c}$ in the steady state. The relaxation time $\tau$ accounts for the decay of the initial polarization $P_{0}$ and also for the evolution of the polarization when the applied bias is changed. In fact, as it will be shown in the following, the current component $I_{c}^{(n l)}=\mathcal{A} \times \partial P_{n l} / \partial t$ has a crucial role in producing the hysteretic effects, representing a significant part of the measured current I:

$$
I=I_{p h}-I_{d}-I_{s h}-I_{c}
$$

where $I_{d}$ is the diode current, $I_{s h}$ accounts for recombinations and $I_{c}=I_{c}^{(l)}+I_{c}^{(n l)}$, with typically negligible linear component $I_{c}^{(l)}$ due to the small geometrical capacitance $C_{0}$. However, depending on the pre-poling conditions $I_{c}^{(n l)}$ can reach magnitudes similar to the measured current [30].

Another version of DEM has been subsequently proposed by Ravishankar et. al. 31 in terms of a relaxation kinetic constant determined by ion displacement, by reformulating Eq. (2) for the surface polarization voltage [Eq. (6) therein]. Both versions of DEM consistently reproduced experimental $\mathrm{NH}$, including the dependence on the bias scan rate and the current overshoot in the reverse scan observed in several experimental studies [5, 18, 32], reproduced only in part by drift-diffusion based models 28]. The overshoot is consistent with a relaxation time of the polarization or charge accumulated at interfaces 30, 31 at the beginning of the reverse scan measurement, indicating a relation between the pre-poling bias and the initial polarization. Furthermore, the dependence of the hysteresis on the bias scan rate is consistently described by the DEM: the hysteresis is diminished at very low and very high bias scan rates, being significant at intermediate ones; importantly, the short circuit current is enhanced with the increase of the bias scan rate.

It is worth noting that a standard constant capacitance model cannot explain the current overshoot, nor can account for the diminished hysteresis at very high scan rates, unless a vanishing dielectric constant for rapidly varying fields is assumed. Moreover, it predicts only the normal hysteresis behavior.

\section{MEASUREMENT PROTOCOL}

As already mentioned in other papers [33, 34], in order to obtain reproducible measurements, it is important to adopt a well defined measurement protocol. We consider three steps: 1 - the stabilization of the open circuit bias $V_{o c}, 2$ - the solar cell pre-conditioning phase by pre-poling under 1 sun illumination, and 3 - the actual $\mathrm{J}-\mathrm{V}$ measurement consisting of a single reverse-forward bias scan starting from the open circuit bias to shortcircuit and back. Before each pre-conditioning phase, the solar cell is kept in open circuit under illumination until the steady state is achieved, corresponding to stabilized $V_{o c}$ and zero current. In this way we reinitialize as far as possible the current state of the solar cell, which may be affected in successive measurements. We choose the open circuit condition for the stabilization phase, instead of the short-circuit, to avoid as much as possible the solar cell deterioration. Pre-poling with illumination is achieved by applying a constant bias $V_{p o l}$ for a time $t_{p o l}$. For $V_{p o l}>V_{o c}$ we call the sample over-polarized, 

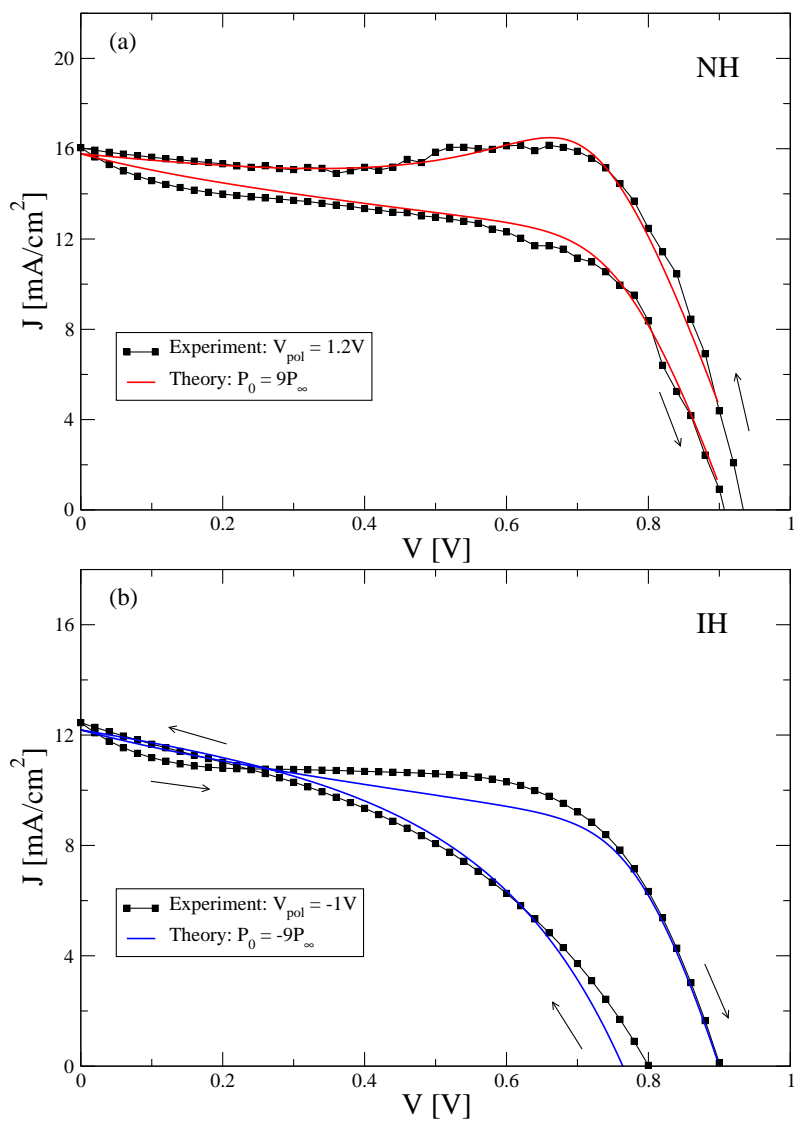

FIG. 2. (a) Calibration of the simulated J-V characteristics using the measured data of a pre-poled sample, at $V_{\text {pol }}=$ $1.2 \mathrm{~V}$ for $t_{\text {pol }}=30 \mathrm{~s}$, exhibiting $\mathrm{NH}$, at a bias scan rate of 20 $\mathrm{mV} / \mathrm{s}$. The corresponding initial polarization is $P_{0}=9 P_{\infty}$. (b) J-V characteristics of the same sample pre-poled at $V_{\text {pol }}=$ $-1 V$ and the resulting simulated $\mathrm{J}-\mathrm{V}$ characteristics, obtained with the same parameters, while changing $P_{0}=-9 P_{\infty}$ and a smaller $I_{p h}=1.11 \mathrm{~mA}$. The IH is present for $0.23 V<V<$ $V_{o c}$, while $\mathrm{NH}$ is obtained for $V<0.23 \mathrm{~V}$.

for $0<V_{\text {pol }}<V_{o c}$ under-polarized, and for $V_{\text {pol }}<0$ reversely polarized. Regarding the actual measurement, instead of performing individual forward and reverse bias scans, the single step reverse-forward bias scan ensures a better control over the sample pre-poling, eliminating potential pre-poling differences in the two separate scans.

\section{RESULTS AND DISCUSSION}

Results and discussion. In the following we discuss the $\mathrm{NH}$ and $\mathrm{IH}$ induced by different pre-poling regimes, the measurements being consistently reproduced by the DEM calculations. The samples are prepared in the standard $\mathrm{FTO} / \mathrm{TiO}_{2} / \mathrm{CH}_{3} \mathrm{NH}_{3} \mathrm{PbI}_{3-x} \mathrm{Cl}_{x} /$ spiroOMeTAD/Au configuration, with $x=0.4$, [26] as detailed in the Supporting Information (SI). More details, including the physical properties and time evolution of similar samples, are described in Ref. [26]. First, we cali- brate the equivalent circuit elements with a measured $\mathrm{J}-\mathrm{V}$ characteristics showing $\mathrm{NH}$, performed at a bias scan rate of $20 \mathrm{mV} / \mathrm{s}$, with positive pre-poling bias $V_{\text {pol }}=1.2 \mathrm{~V}$ for $t_{p o l}=30 \mathrm{~s}$. With this choice, the current overshoot is present in the reverse characteristics and this marked feature enables a more accurate extraction of the model parameters [30, 31]. The simulated J-V characteristics of the over-polarized sample is depicted in Fig. 2(a) matching closely the detailed features of the experimental data. The obtained DEM parameters are: the photogenerated current $I_{p h}=1.42 \mathrm{~mA}$, the series resistance $R_{s}=95 \Omega$, the shunt resistance $R_{s h}=3 k \Omega$, the diode ideality factor $n=1.53$ and saturation current $I_{s}=0.1 \mathrm{pA}$. The relative dielectric permittivity $\epsilon_{r}=100$ yields a quite small geometrical capacitance $C_{0}$ and its influence over the $\mathrm{J}-\mathrm{V}$ characteristics is negligible in comparison with the nonlinear polarization component. The steady state polarization at the open circuit is $P_{\infty}=18 \mathrm{mC} / \mathrm{cm}^{2}$, corresponding to a stabilized $V_{o c}=0.9 \mathrm{~V}$. The relaxation time $\tau=9 \mathrm{~s}$ fits well with the preliminary indication about the magnitude of the characteristic times involved. The assumed initial polarization $P_{0}=9 P_{\infty}$ corresponds to the pre-poling stage performed under illumination at 1.2 $\mathrm{V}$ for $30 \mathrm{~s}$. The device active area is $\mathcal{A}=0.09 \mathrm{~cm}^{2}$, which is also the illuminated area.

By switching the initial polarization $P_{0}$ to negative values, J-V characteristics with mixed or completely inverted hysteresis are observed. It is quite remarkable that changing only one parameter, $P_{0}=-9 P_{\infty}$, the shape of the inverted hysteresis resembles a measured characteristics with $V_{p o l}=-1 \mathrm{~V}$ for $30 \mathrm{~s}$ at the same bias scan rate, apart from the diminished short circuit current obtained in the measurement. Taking into account $I_{p h}=1.11 \mathrm{~mA}$ the simulated $\mathrm{J}-\mathrm{V}$ characteristics overlaps quite well with the experimental data, as indicated in Fig. 2(b). Our test case shows a mostly inverted hysteresis, with a crossing point in the forward and reverse characteristics at $\sim 0.23$ $\mathrm{V}$. The decrease of $I_{s c}$ at negative pre-poling was systematically observed in repeated measurements, starting from lower $V_{p o l}$ values to higher ones and in reverse order, which will be discussed later. Apart from this poling effect, a temporary aging of the cell is observed after repeated bias cycling, manifested by an overall decrease of the current in both forward and reverse characteristics. Typically, the samples recover in one day in dark and inert atmosphere conditions.

Both hysteretic effects can be explained by analyzing the time evolution of the non-linear polarization $P_{n l}(t)$, depicted in Fig. 3. In the case of NH, $P_{n l}(t=0)=P_{0} \gg$ $P_{\infty}$ is positive and decays rather fast in a time interval of the order of $\tau$ in the beginning of the reverse scan. Subsequently, $P_{n l}$ follows the bias dependent steady state polarization $P_{n l, \infty}\left(U_{c}(t)\right)$ [Eq. (2)] as the bias is further decreased in reverse and then increased in the forward regime. On the other hand, $\mathrm{IH}$ is produced by negative poling, yielding a negative initial polarization, $P_{0}<0$. The measurement, however, starts from $V_{o c}$ and $P_{n l}$ is driven into positive region on the reverse scan, reaching 

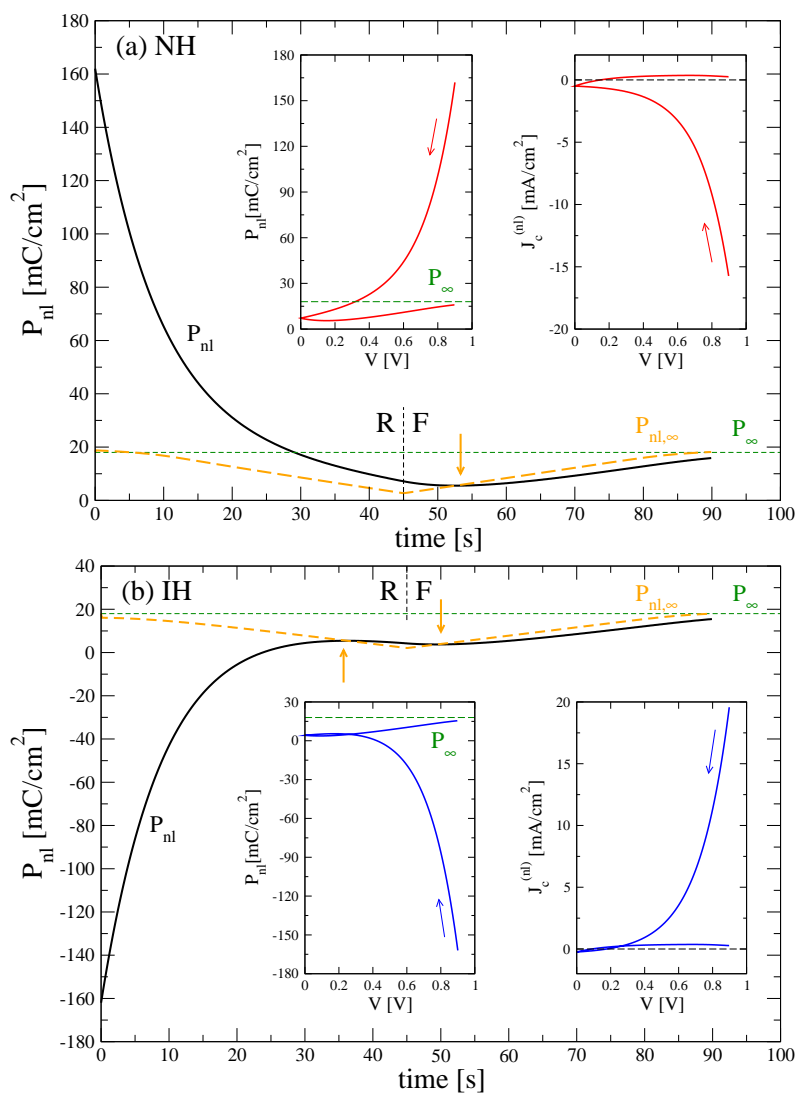

FIG. 3. Time evolution of the non-linear polarization $P_{n l}$ for $\mathrm{NH}$ (a) and $\mathrm{IH}(\mathrm{b})$, corresponding to J-V characteristics shown in Fig. 2 The vertical arrows mark the crossing points between the bias dependent steady state polarization $P_{n l, \infty}$ and the current polarization $P_{n l}$. The insets show the bias dependence of $P_{n l}$ and $J_{c}^{(n l)}=\partial P_{n l} / \partial t$. The vertical dashed lines mark the separation between the reverse and forward regimes.

a maximum, and then decreasing towards the zero-bias state. As $\tau$ is here smaller than the total time of the reverse scan, the behavior of $P_{n l}$ in the forward scan is similar for both $\mathrm{IH}$ and NH. The condition $P_{n l, \infty}=P_{n l}$ is correlated either with a minimum or a maximum for $P_{n l}(t)$. As already indicated, the $J_{c}^{(n l)}$ current component is quantitatively significant and plays a determinant role in the features on the J-V characteristics. Depending on its sign the value of the measured current can be enhanced or diminished according to Eq. (3), influencing the determined PCE value.

To further investigate the transition between $\mathrm{NH}$ and $\mathrm{IH}$, we measured J-V characteristics with several prepoling voltages, between $-1.5 \mathrm{~V}$ to $1.2 \mathrm{~V}$, as shown in Fig. 4 together with the calculated J-V characteristics with suitably chosen initial polarizations $P_{0}$. A crossing point between the reverse and forward characteristics appears and it moves systematically towards lower biases as $P_{0}\left(\right.$ or $\left.V_{\text {pol }}\right)$ decreases. This behavior was theoretically predicted before, in Ref. [30], as an under-polarization
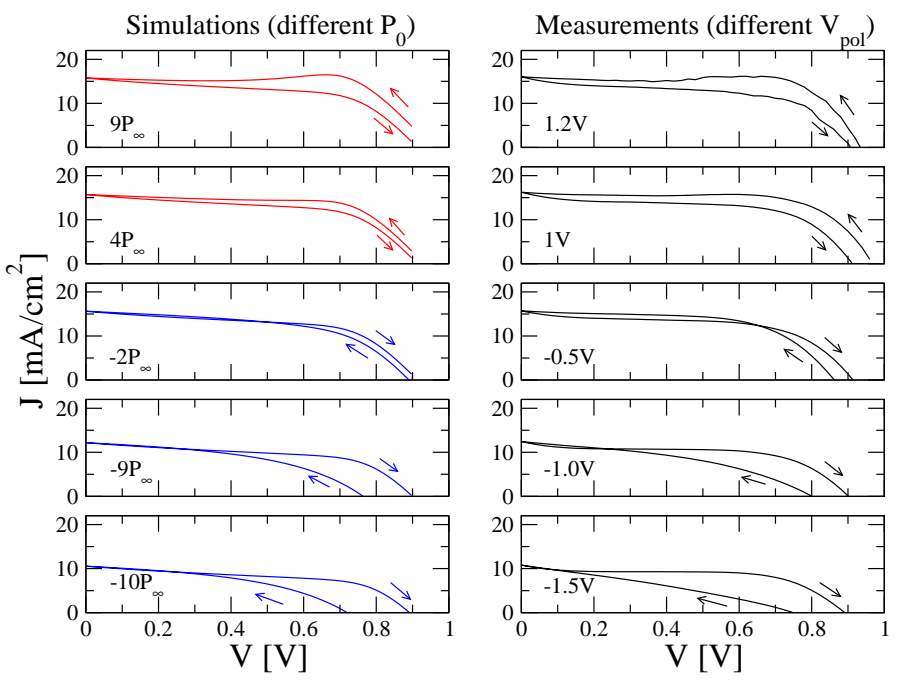

FIG. 4. Normal and inverted hysteresis, obtained by changing the pre-poling bias $V_{\text {pol }}$ (measurements, right panel) and, correspondingly, the initial polarization $P_{0}$ (simulations, left panel), for a bias scan rate of $20 \mathrm{mV} / \mathrm{s}$.

regime $\left(0<P_{0}<P_{\infty}\right)$. On the other hand, the crossing was observed experimentally [26] on aged samples with Ag counter electrodes, using a forward-reverse scan protocol starting from $-0.1 \mathrm{~V}$, which may be the effect of a small negative bias pre-poling, although not intentionally performed. The crossing of separately measured forward and reverse characteristics was also observed [31]. As already indicated, a particular feature is the decrease of the $I_{s c}$ found in the measured $\mathrm{J}-\mathrm{V}$ characteristics, as negative poling increases in absolute value. Based on this experimental observation and the fact that $R_{s h}$ is relatively large, which implies $I_{s c} \approx I_{p h}$, and assuming a relaxation time independent on the poling bias, we adjust the $I_{p h}$ current to $1.11 \mathrm{~mA}\left(P_{0}=-9 P_{\infty}\right)$ and $0.96 \mathrm{~mA}$ $\left(P_{0}=-10 P_{\infty}\right)$ for $V_{p o l}=-1 \mathrm{~V}$ and $-1.5 \mathrm{~V}$, respectively. This may be explained by iodine migration during the poling time, as negative iodine ions tend to accumulate at the $\mathrm{TiO}_{2}$ mesoporous layer and the induced electric field reduces electron collection during the subsequent measurement [25]. By contrast, when positive poling is applied the electric field induced by ions is favorable to electron extraction and $I_{s c}$ does not change significantly.

We focus next on the bias scan rate dependence of the IH. For the NH we obtained a consistent correspondence between the measurements and DEM [30]: the NH is widest at intermediate bias scan rates and $I_{s c}$ increases at higher rates. This behavior was explained in terms of time evolution of non-linear polarization $P_{n l}$ and the current component $J_{c}^{(n l)}=\partial P_{n l} / \partial t$ introduced in Eq. (2), as also shown (for comparison to IH) in Fig. S1 of SI. The simulated and measured J-V characteristics showing IH are presented in Fig. 5. In the experiment we considered $V_{\text {pol }}=-1.4 \mathrm{~V}$, which corresponds in simulations to $P_{0}=-9.5 P_{\infty}$. At this stage the sample is slightly aged, as one may notice from the $20 \mathrm{mV} / \mathrm{s}$ scan, having 

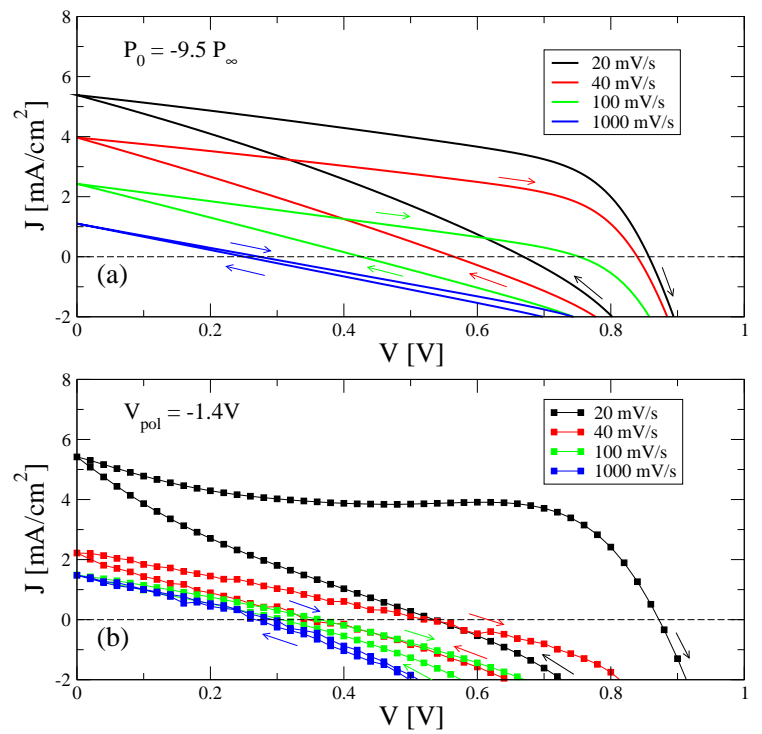

FIG. 5. Inverted hysteresis under varying the bias scan rate: (a) simulated $\mathrm{J}-\mathrm{V}$ characteristics for bias scan rates in the range $20-1000 \mathrm{mV} / \mathrm{s}$, with $P_{0}=-9.5 P_{\infty}$; (b) measured $\mathrm{J}-\mathrm{V}$ characteristics, showing the decreasing of $I_{s c}$ with increasing the bias scan rate.

a smaller $I_{s c}$ compared to bias pre-poling measurements. We therefore consider $I_{p h}=0.6 \mathrm{~mA}$ and an increased $\tau=30 \mathrm{~s}$ and reproduce the sequence of experimental $\mathrm{J}-\mathrm{V}$ characteristics with different bias scan rates. As in the case of NH at very fast bias scans, the hysteresis amplitude is diminished. However, the behavior of $I_{s c}$ is opposite: it becomes smaller as the scan rate increases. At very fast bias scan rates there is little variation in $P_{n l}$, as it remains close to $P_{0}$, and $\partial P_{n l} / \partial V$ becomes small in both forward and reverse scans, reducing thus the hysteresis of both $\mathrm{NH}$ and IH. In contrast, at short-circuit, the calculated current $J_{c}^{(n l)}=\partial P_{n l} / \partial t$ has a larger magnitude for $\mathrm{NH}$ at higher bias scan rates, and the opposite occurs for IH (Fig. S1). This accounts for both measured and calculated decrease in $I_{s c}$ in IH conditions (Figs. S2 and [33). We also note that $\partial P_{n l} / \partial t<0$ for $\mathrm{NH}$ and $\partial P_{n l} / \partial t>0$ for $\mathrm{IH}$, which is the reason for the crossing of the forward and reverse characteristics. We therefore conclude that the pre-poling bias is a determinant switching factor from $\mathrm{NH}$ to IH, which become two forms of hysteresis previously considered independent.

The dynamic hysteresis creates difficulties for an accurate determination of the solar cell PCE. Figure 6 shows $\mathrm{NH}\left(P_{0}=9 P_{\infty}\right), \mathrm{IH}\left(P_{0}=-9 P_{\infty}\right)$ cases and a minimal polarization case $\left(P_{0}=P_{\infty}\right)$, for a $20 \mathrm{mV} / \mathrm{s}$ bias scan rate, and the steady state solution $\left(P_{\infty}=0\right)$, in the bias range $\left[0, V_{o c}\right]$. The PCE varies drastically in forward and reverse scans of both $\mathrm{NH}$ and $\mathrm{IH}$, with a large deviation in the reverse scans compared to the steady state PCE: $27 \%$ overestimation for $\mathrm{NH}$ and $48 \%$ underestimation for IH. In the forward scan of the NH we find a deviation of only $2.3 \%$, while for IH we obtain a $28 \%$ smaller PCE.

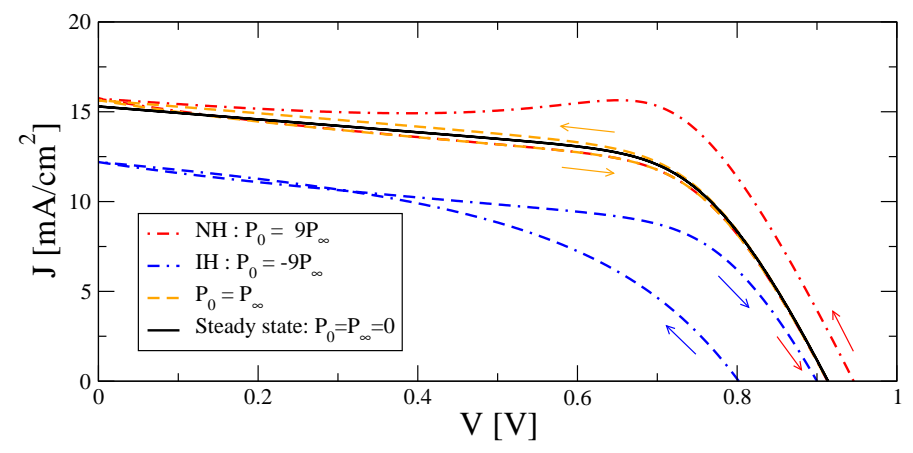

FIG. 6. Analyzing the PCEs under different poling conditions in comparison with the reference value obtained from the static J-V characteristics: NH:10.75\%, IH:4.47\%, $P_{\infty}: 8.54 \%$ in reverse bias scan and $\mathrm{NH}: 8.24 \%$, IH:6.12\% $P_{\infty}: 8.23 \%$ in forward scan; the steady state yields the PCE value of $8.44 \%$.

However in the minimal polarization case, where the reverse scan starts at $V_{o c}$ determined before the actual measurement, only a small hysteresis is found, as $P_{\infty} \neq 0$. This case provides the most accurate description of the steady state, with a deviation of less than $3 \%$ for both forward and reverse scans. Decreasing the bias scan rate would reduce the hysteresis loop even further. However, in practical measurements, for a reasonable measurement time, it is very important to determine firstly $V_{o c}$, in order to avoid over-polarization. Secondly, another recommendation within the proposed measurement protocol is taking a continuous reverse-forward cycle, which is essential for removing an unintended initial polarization, e.g. by applying a reverse scan ending into negative voltage followed by a separate forward scan after an arbitrary time interval. A waiting time between reverse and forward bias scans influence significantly the second (forward) scan, as it is further detailed in Fig. S4 (SI), for an IH test case. Therefore we emphasize the importance of a careful consideration of the cell dynamic polarization. Our three-step protocol allows a systematic evaluation of such hysteretic phenomena, but also provides a realistic determination of the PCE as the initial polarization is minimized.

\section{CONCLUSIONS}

To conclude, we investigated the dynamic hysteresis of standard perovskite solar cells with controlled preconditioning. By varying the pre-poling bias, both normal and inverted hysteresis are found for the same sample and the measured $\mathrm{J}-\mathrm{V}$ characteristics are closely reproduced. The main findings of the present study are:

1. The NH and IH can be obtained by pre-poling the solar cell with bias $V_{p o l}>V_{o c}$ and $V_{p o l}<0$, respectively, and a mixed hysteresis is typically observed for $0<V_{p o l}<V_{o c}$.

2. The DEM reproduces the two hysteretic behaviors 
by changing only the initial polarization parameter, which is related to experimental quantities $V_{p o l}$ and $t_{p o l}$.

3. At higher bias scan rates the short circuit current $I_{s c}$ is enhanced for $\mathrm{NH}$ and lowered for $\mathrm{IH}$, consistently explained by DEM.

4. The observed $\mathrm{NH} / \mathrm{IH}$ behavior reveals once again the importance of controlling the solar cell preconditioning, particularly the bias pre-poling; the state induced by a previous measurement may inadvertently affect the next one; the designed threestep protocol, including stabilization, pre-poling and measurement is afforded for the reliability and reproducibility of the experimental data, being especially important for a correct PCE determina- tion.

5. The presence of both NH and IH unifies apparently contradictory results reported in recent papers. Our results indicate the presence of both normal and the inverted hysteresis in the same device if the required pre-poling conditions can be achieved.

\section{ACKNOWLEDGMENTS}

The research leading to these results has received funding from EEA Financial Mechanism 2009-2014 under the project contract no 8SEE/30.06.2014.
[1] H. J. Snaith, A. Abate, J. M. Ball, G. E. Eperon, T. Leijtens, N. K. Noel, S. D. Stranks, J. T.-W. Wang, K. Wojciechowski, and W. Zhang, J. Phys. Chem. Lett. 5, 1511 (2014).

[2] A. Dualeh, T. Moehl, N. Tétreault, J. Teuscher, P. Gao, M. K. Nazeeruddin, and M. Grätzel, ACS Nano 8, 362 (2014).

[3] N. K. Elumalai and A. Uddin, Sol. Energy Mater. Sol. Cells 157, 476 (2016).

[4] D. A. Egger, E. Edri, D. Cahen, and G. Hodes, J. Phys. Chem. Lett. 6, 279 (2015).

[5] W. Tress, N. Marinova, T. Moehl, S. M. Zakeeruddin, M. K. Nazeeruddin, and M. Gratzel, Energy Environ. Sci. 8, 995 (2015).

[6] M. De Bastiani, G. Dell'Erba, M. Gandini, V. D'Innocenzo, S. Neutzner, A. R. S. Kandada, G. Grancini, M. Binda, M. Prato, J. M. Ball, M. Caironi, and A. Petrozza, Adv. Energy Mater. 6, 1501453 (2016).

[7] E. Christopher, J. M. Frost, P. R. F. Barnes, B. C. O'Regan, A. Walsh, and M. S. Islam, Nature Commun. 6, 7497 (2015).

[8] Y. Zhao, C. Liang, H. Zhang, D. Li, D. Tian, G. Li, X. Jing, W. Zhang, W. Xiao, Q. Liu, F. Zhang, and Z. He, Energy Environ. Sci. 8, 1256 (2015).

[9] B. Chen, M. Yang, X. Zheng, C. Wu, W. Li, Y. Yan, J. Bisquert, G. Garcia-Belmonte, K. Zhu, and S. Priya, J. Phys. Chem. Lett. 6, 4693 (2015).

[10] Y. Shao, Z. Xiao, C. Bi, Y. Yuan, and J. Huang, Nature Commun. 5, 5784 (2014).

[11] J. Xu, A. Buin, A. H. Ip, W. Li, O. Voznyy, R. Comin, M. Yuan, S. Jeon, Z. Ning, J. J. McDowell, P. Kanjanaboos, J.-P. Sun, X. Lan, L. N. Quan, D. H. Kim, I. G. Hill, P. Maksymovych, and E. H. Sargent, Nature Commun. 6, 7081 (2015).

[12] J. Wei, Y. Zhao, H. Li, G. Li, J. Pan, D. Xu, Q. Zhao, and D. Yu, J. Phys. Chem. Lett. 5, 3937 (2014).

[13] H.-W. Chen, N. Sakai, M. Ikegami, and T. Miyasaka, J. Phys. Chem. Lett. 6, 164 (2015).

[14] J. M. Frost, K. T. Butler, and A. Walsh, APL Mater. 2, 081506 (2014).

[15] J. M. Frost, K. T. Butler, F. Brivio, C. H. Hendon, M. van Schilfgaarde, and A. Walsh, Nano Lett. 14, 2584
(2014).

[16] E. J. Juarez-Perez, R. S. Sanchez, L. Badia, G. GarciaBelmonte, Y. S. Kang, I. Mora-Sero, and J. Bisquert, J. Phys. Chem. Lett. 5, 2390 (2014).

[17] O. Almora, I. Zarazua, E. Mas-Marza, I. Mora-Sero, J. Bisquert, and G. Garcia-Belmonte, J. Phys. Chem. Lett. 6, 1645 (2015).

[18] R. S. Sanchez, V. Gonzalez-Pedro, J.-W. Lee, N.-G. Park, Y. S. Kang, I. Mora-Sero, and J. Bisquert, J. Phys. Chem. Lett. 5, 2357 (2014).

[19] B. Wu, K. Fu, N. Yantara, G. Xing, S. Sun, T. C. Sum, and N. Mathews, Adv. Energy Mater. 5, 1500829 (2015).

[20] G. A. Sepalage, S. Meyer, A. Pascoe, A. D. Scully, F. Huang, U. Bach, Y.-B. Cheng, and L. Spiccia, Adv. Funct. Mater. 25, 5650 (2015).

[21] V. W. Bergmann, S. A. L. Weber, F. J. Ramos, M. K. Nazeeruddin, M. Grätzel, D. Li, A. L. Domanski, I. Lieberwirth, S. Ahmad, and R. Berger, Nature Commun., 5001 (2014).

[22] X. Cao, Y. Li, C. Li, F. Fang, Y. Yao, X. Cui, and J. Wei, J. Phys. Chem. C 120, 22784 (2016).

[23] C. Zhao, B. Chen, X. Qiao, L. Luan, K. Lu, and B. Hu, Adv. Energy Mater. 5, 1500279 (2015).

[24] W. Tress, J. P. Correa Baena, M. Saliba, A. Abate, and M. Graetzel, Adv. Energy Mater. 6, 1600396 (2016).

[25] D. A. Jacobs, Y. Wu, H. Shen, C. Barugkin, F. J. Beck, T. P. White, K. Weber, and K. R. Catchpole, Phys. Chem. Chem. Phys. 19, 3094 (2017).

[26] C. Besleaga, L. E. Abramiuc, V. Stancu, A. G. Tomulescu, M. Sima, L. Trinca, N. Plugaru, L. Pintilie, G. A. Nemnes, M. Iliescu, H. G. Svavarsson, A. Manolescu, and I. Pintilie, J. Phys. Chem. Lett. 7, 5168 (2016).

[27] S. van Reenen, M. Kemerink, and H. J. Snaith, J. Phys. Chem. Lett. 6, 3808 (2015).

[28] G. Richardson, S. E. J. O'Kane, R. G. Niemann, T. A. Peltola, J. M. Foster, P. J. Cameron, and A. B. Walker, Energy Environ. Sci. 9, 1476 (2016).

[29] K. Seki, Appl. Phys. Lett. 109, 033905 (2016).

[30] G. A. Nemnes, C. Besleaga, A. G. Tomulescu, I. Pintilie, L. Pintilie, K. Torfason, and A. Manolescu, Sol. Energy Mater. Sol. Cells 159, 197 (2017). 
[31] S. Ravishankar, O. Almora, C. Echeverria-Arrondo, E. Ghahremanirad, C. Aranda, A. Guerrero, F. FabregatSantiago, A. Zaban, G. Garcia-Belmonte, and J. Bisquert, J. Phys. Chem. Lett. 8, 915 (2017).

[32] S. Meloni, T. Moehl, W. Tress, M. Franckevicius, M. Saliba, Y. H. Lee, P. Gao, M. K. Nazeeruddin, S. M. Zakeeruddin, U. Rothlisberger, and M. Graetzel, Nature Commun., 10334 (2016).
[33] J. A. Christians, J. S. Manser, and P. V. Kamat, J. Phys. Chem. Lett. 6, 852 (2015).

[34] E. Zimmermann, K. K. Wong, M. Müller, H. Hu, P. Ehrenreich, M. Kohlstädt, U. Würfel, S. Mastroianni, G. Mathiazhagan, A. Hinsch, T. P. Gujar, M. Thelakkat, T. Pfadler, and L. Schmidt-Mende, APL Mater. 4, 091901 (2016).

[35] N. Ahn, D. Son, I. Jang, S. M. Kang, M. Choi, and N. Park, J. Am. Chem. Soc. 137, 8698 (2015).

Appendix: Supporting Information

\section{Device fabrication and characterization.}

\section{a) Device fabrication}

We fabricated perovskite solar cells by successive spin-coating deposition of TiO2 thin and meso-porous layers, $\mathrm{CH}_{3} \mathrm{NH}_{3} \mathrm{PbI}_{3-x} \mathrm{Cl}_{x}$ mixed halide perovskite and spiro-OMeTAD on commercial glass/FTO substrate (Solaronix TCO22-7). The compact $\mathrm{TiO}_{2}$ blocking layer of $150 \mathrm{~nm}$ thickness is obtained from bis(acetylacetonate) solution (Aldrich) and annealing at $450{ }^{\circ} \mathrm{C}$ for $30 \mathrm{~min}$. The mesoporous $\mathrm{TiO}_{2}$ film $\left(\mathrm{mp}-\mathrm{TiO}_{2}\right)$, composed of $20 \mathrm{~nm}$ size particles is deposited using a solution of $\mathrm{TiO}_{2}$ commercial paste (Solaronix Ti-Nanoxide N/SP) diluted in ethanol (1:3, weight ratio). The mp- $\mathrm{TiO}_{2}$ structures were first annealed at $150{ }^{\circ} \mathrm{C}$ for $5 \mathrm{~min}$ and then crystallized at $500{ }^{\circ} \mathrm{C}$ for $1 \mathrm{~h}$. The $\mathrm{CH}_{3} \mathrm{NH}_{3} \mathrm{PbI}_{3-x} \mathrm{Cl}_{x}$ mixt halide perovskite is fabricated using the principle of one-step method [35]: a precursor solution containing $369 \mathrm{mg}$ lead iodide, $56 \mathrm{mg}$ lead chloride, $78 \mathrm{mg}$ methyl sulfoxide, $159 \mathrm{mg}$ methyl ammonium iodide (Dysol) and $600 \mathrm{mg}$ dimethylformamide, homogenized for one hour, was spin coated with 2000 $\mathrm{rpm} / 25 \mathrm{~s}$. After $9 \mathrm{~s}$ from the start of the spin cycle $150 \mu \mathrm{l}$ of diethyl ether was dripped on top of the layer, enabling the solvent extraction process to take place. The final perovskite film is obtained after annealing at $65{ }^{\circ} \mathrm{C}$ for $1 \mathrm{~min}$ and $100{ }^{\circ} \mathrm{C}$ for $2 \mathrm{~min}$. All the above described processes were performed in normal laboratory conditions, at $24{ }^{\circ} \mathrm{C}$ and humidity between $30 \%$ and $40 \%$. The spiro-OMeTAD was spin-coated at $1500 \mathrm{rpm}$ for $30 \mathrm{~s}$, in $\mathrm{N}_{2}$ enriched atmosphere, at $24{ }^{\circ} \mathrm{C}$ and humidity less than $10 \%$. The solution used for this deposition was obtained by mixing $80 \mathrm{mg}$ spiro-OMeTAD (Borun Chemical), $28 \mu \mathrm{l}$ 4-tert-butylpyridine and $18 \mu \mathrm{l}$ of bis(trifluoromethane)sulfonimide lithium salt in acetonitrile solution $\left(520 \mathrm{mg} \mathrm{ml}^{-1}\right)$. Gold counter electrodes of $0.09 \mathrm{~cm}^{2}$ area have been deposited by magnetron RF sputtering.

\section{b) Electrical characterization}

The Photocurrent-Voltage $(\mathrm{J}-\mathrm{V})$ characteristics were measured using an Oriel VeraSol-2 Class AAA LED Solar Simulator having AM 1.5 filters and a Keithley 2400 Source Meter. The irradiation intensity was $100 \mathrm{~mW} / \mathrm{cm}^{2}$, calibrated by a Newport standard silicon solar cell 91150. The 1 Sun illumination has been performed through a rectangular aperture of $3 \times 3 \mathrm{~mm}^{2}$ size, of a geometry identical with that of Au counter electrodes. 
2. Simulations and measurements revealing normal hysteresis (NH) or inverted hysteresis (IH), for different bias scan rates and prepoling biases.

a) Simulated $\mathrm{NH}$ and $\mathrm{IH}$ for different bias scan rates

NH
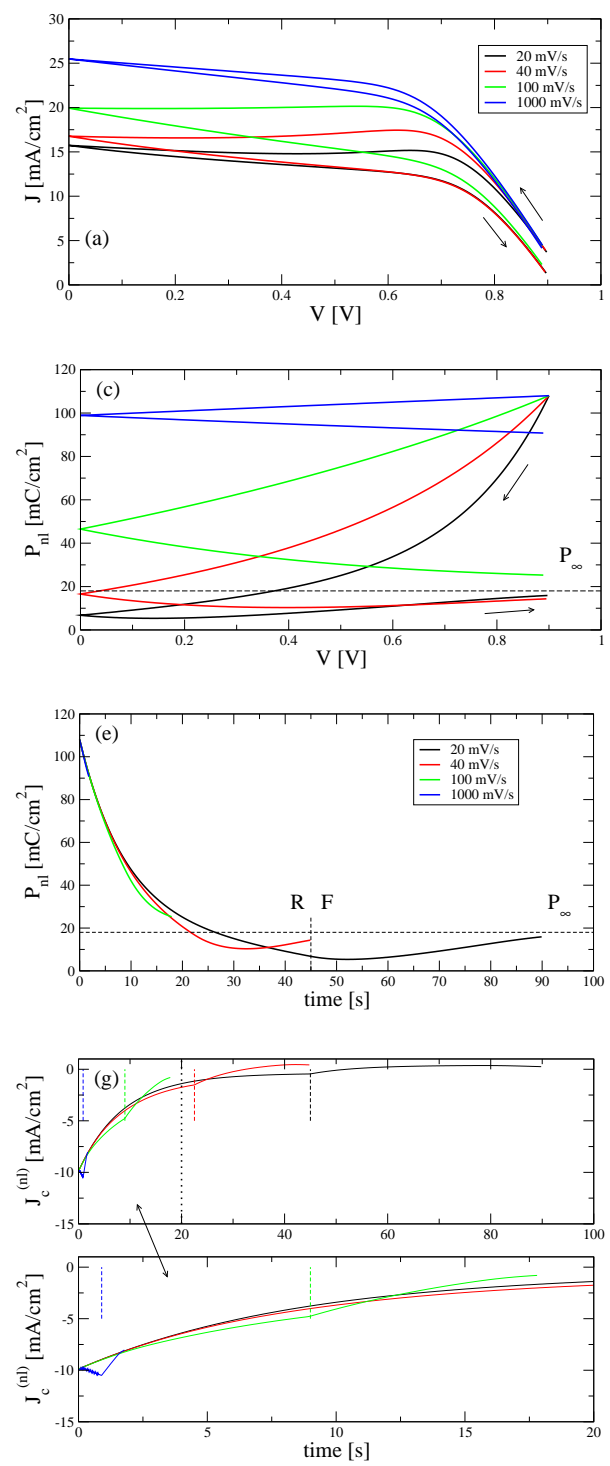

IH
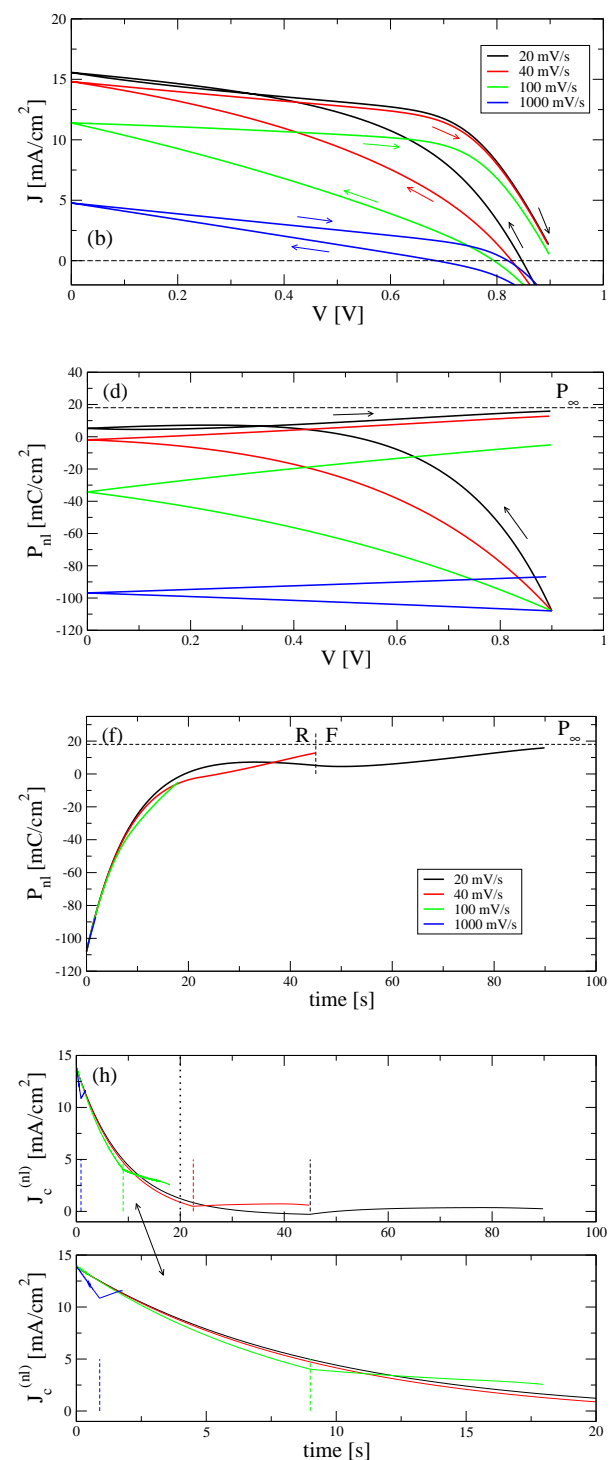

FIG. S1. Simulated reverse-forward NH and IH behavior for different bias scan rates, with pre-poling $P_{0}=6 P_{\infty}$ and $P_{0}=-6 P_{\infty}$, while all other parameters are the same as the ones used in Fig. 2(a). (a,b) J-V characteristics. (c,d) Non-linear polarization $P_{n l}$ as a function of applied bias. Time evolution of $P_{n l}(\mathrm{e}, \mathrm{f})$ and $J_{c}^{(n l)}(\mathrm{g}, \mathrm{h})$. At short-circuit (marked by dashed lines) $J_{c}^{(n l)}$ is increasing in absolute value with the scan rate. 
b) Experimental NH and IH induced by bias pre-poling
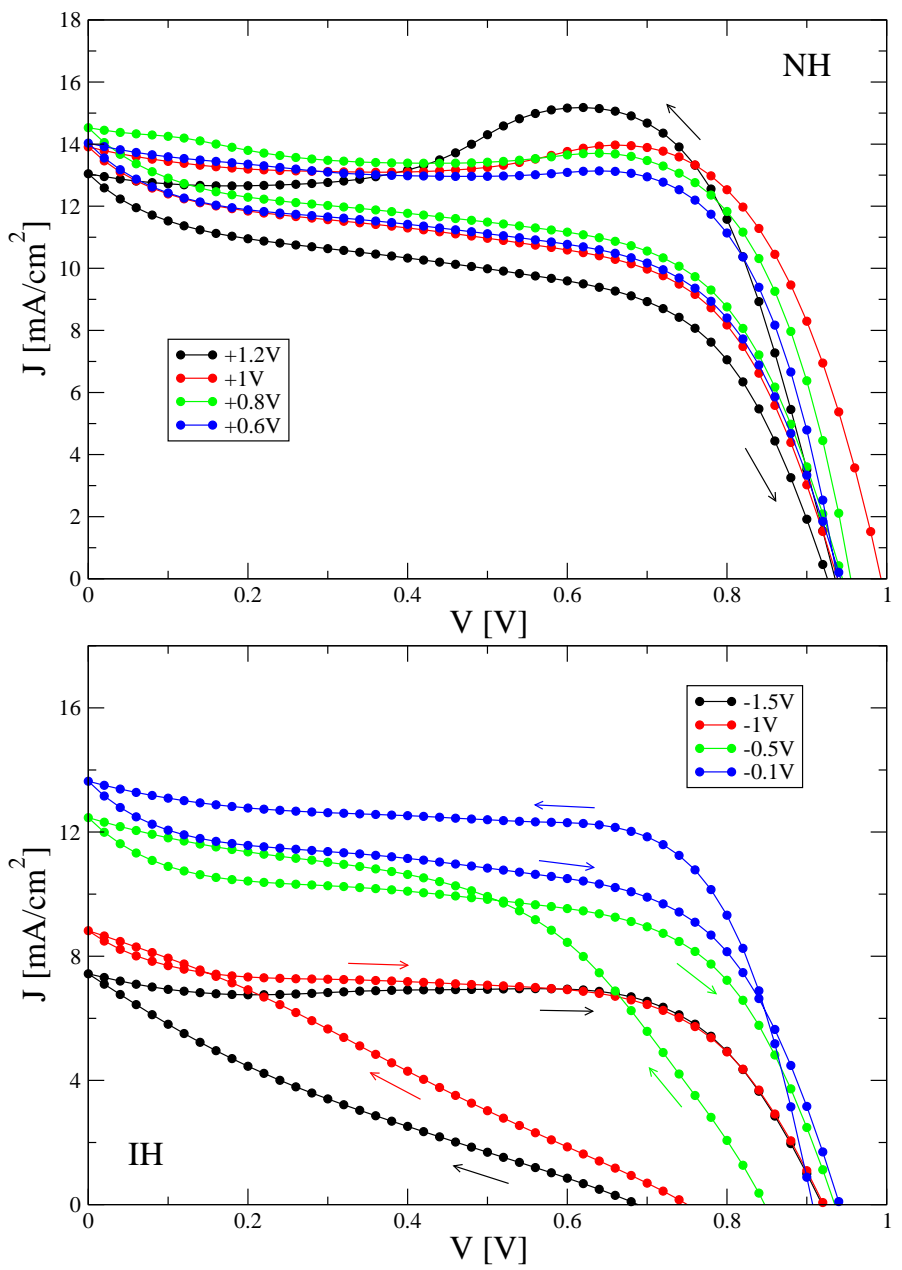

FIG. S2. Experimental J-V characteristics showing NH and IH under pre-poling conditions: NH is obtained for positive prepoling biases $V_{p o l}=0.6 \mathrm{~V}, 0.8 \mathrm{~V}, 1.0 \mathrm{~V}, 1.2 \mathrm{~V}$, IH is obtained for $V_{p o l}=-1.5 \mathrm{~V}$, while the mixed hysteresis characterized by a crossing point is found for $V_{p o l}=-0.1 \mathrm{~V},-0.5 \mathrm{~V},-1 \mathrm{~V}$. The poling time is in each case $t_{p o l}=30 \mathrm{~s}$. 
c) Experimental NH and IH - influence of the bias scan rate
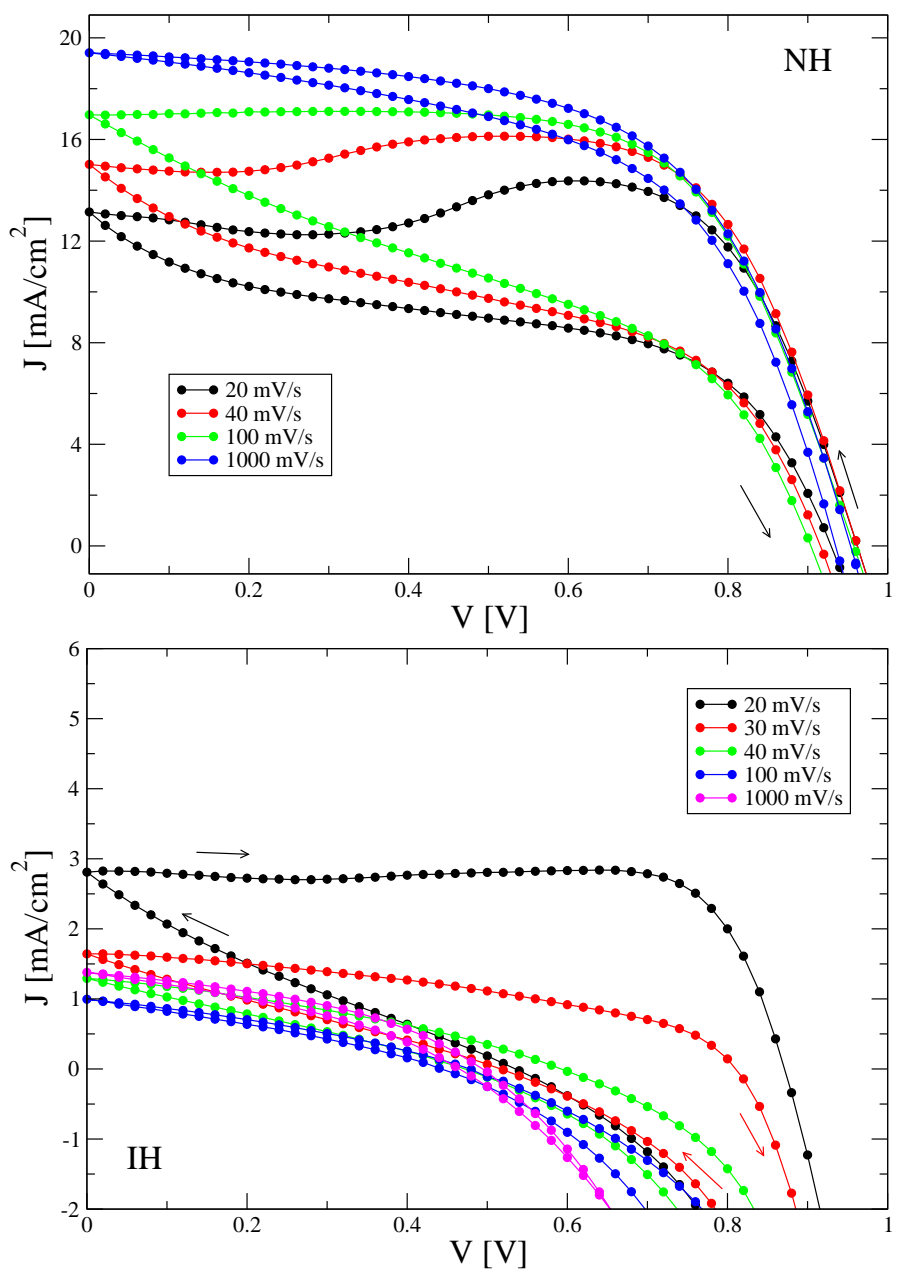

FIG. S3. Experimental J-V characteristics showing $\mathrm{NH}$ and $\mathrm{IH}$ for different scan rates, in the range $20-1000 \mathrm{mV} / \mathrm{s}$. The samples exhibiting $\mathrm{NH}$ are pre-poled at $V_{\text {pol }}=1.2 \mathrm{~V}$, while $\mathrm{IH}$ characteristics are obtained for $V_{\text {pol }}=-1.5 \mathrm{~V}$, with $t_{p o l}=30 \mathrm{~s}$. 
3. Influence of the waiting time at short-circuit, between reverse and forward scan, for the case of inverted hysteresis.
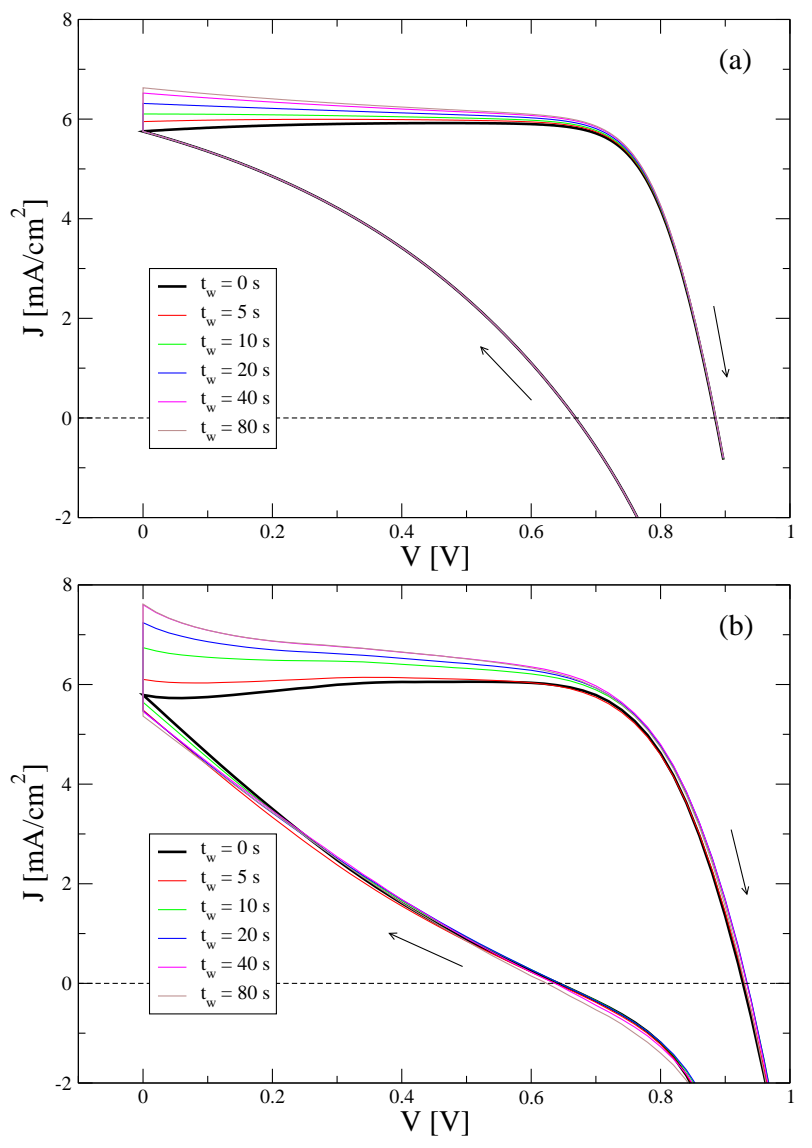

FIG. S4. The influence of the waiting time $t_{w}$ spent at open circuit, between the reverse and forward scans $(20 \mathrm{mV} / \mathrm{s})$ : simulation (a) and experiment (b). In the simulation, the relaxation time considered is $\tau=20 \mathrm{~s}$, while the experimental pre-poling conditions are $V_{p o l}=-1.5 \mathrm{~V}$ and $t_{p o l}=30 \mathrm{~s}$. Other parameters which are modified for this particular sample with respect to calibration set are: $R_{s h}=30 \mathrm{k} \Omega$ and $I_{p h}=0.6 \mathrm{~mA}$, while the initial polarization is $P_{0}=-12 P_{\infty}$. Beyond $t_{w}=40$ $\mathrm{s}$, the $\mathrm{J}-\mathrm{V}$ characteristics tend to overlap. 